\title{
A Comparison of Experimentally Measured and Theoretically Cal- culated Velocity Fields in a Water Model of an Argon Stirred Ladle
}

\author{
O. J. ILEGBUSI, J. SZEKELY, ${ }^{1)}$ Manabu IGUCHI, ${ }^{2)}$ Hiroaki TAKEUCHI') and Zen-ichiro MORITA ${ }^{2)}$ \\ Department of Mechanical Engineering, Northeastern University, Boston, MA 02115, U.S.A. \\ 1) Department of Materials \\ Science and Engineering, Massachusetts Institute of Technology, Cambridge, MA 02139, U.S.A. \\ 2) Department of \\ Materials Science and Processing, Chemical Processing and Metallurgy Group, Osaka University, Osaka, 565 Japan.
}

(Received on November 16, 1992; accepted in final form on January 22, 1993)

\begin{abstract}
A model is developed to calculate the multi-phase flow produced by the bubbling of air into water. Two turbulence models are employed to represent the turbulence field namely, the $k-\varepsilon$ model and an anisotropic eddy viscocity (algebraic stress) model. The predicted mean flow and turbulence parameters compare well with the experimental data.
\end{abstract}

KEY WORDS: two-phase flow; argon-stirred ladle; numerical simulation; velocity measurement; anisotropic model of turbulence.

\section{Introduction}

In recent years there has been a growing interest in the quantitative representation of flow phenomena in ladles or metallurgical reactors, which are agitated by a jet plume of the type sketched in Fig. 1. These problems are of relevance to a range of ladle metallurgy operations as well as to the various direct iron and steelmaking systems that are currently contemplated.

In the early work the shape of the gas plume was specified and under these conditions calculations could be quite readily performed. However the prediction of the plume shape is of major importance, especially if one wishes to extrapolate from aqueous to metallic systems. Our understanding of these systems has been substantially advanced by three sets of recent publications. ${ }^{1-5)}$ In one group Brimacombe and co-workers ${ }^{1,2)}$ have re-

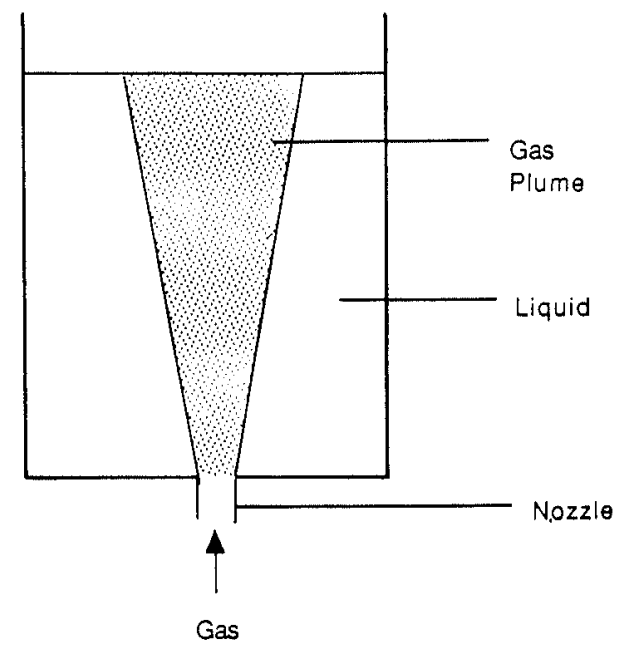

Fig. 1. A schematic sketch of a gas-plume circulation system. ported on very carefully conducted experimental measurements describing the plume shape and consistency in a water pool, agitated by an injected air stream. In another group of papers Iguchi et $a l^{3,4)}$ reported on carefully conducted velocity and turbulence measurements in a water pool agitated by an injected air stream. Finally Ilegbusi and Szekely ${ }^{5)}$ were able to use mathematical modelling techniques to predict the plume shapes from first principles and these predictions were found to be in good agreement with the results reported by Brimacombe. However, up to the present a really critical comparison of the theoretical predictions and experimental measurements, regarding the velocity fields and turbulence levels has not yet been undertaken. Such a comparison would be highly desirable because the knowledge of the turbulence and the velocity fields is an essential pre-requisite to tackling the heat transfer, mass transfer and kinetics problems that are of the ultimate interest to the ladle metallurgists and steelmakers.

In the present paper we seek to combine the earlier mathematical modelling efforts by Ilegbusi and Szekely and the experimental measurements of Iguchi et al. in order to test these theoretical predictions against experimental measurements.

\section{Statement of the Problem}

We shall consider a liquid pool, which is agitated by an injected gas stream, such as sketched in Fig. 1. Our problem is to compute the plume shape and the velocity fields and to compare these predictions to the measurements.

\subsection{The Mathematical Formulation}

The mathematical formulation has been fully described in a recent publication and only a brief outline will be 
Table 1. Exchange coefficients and source terms.

\begin{tabular}{llll}
\hline$\phi_{j}$ & $\Gamma_{m}$ & \multicolumn{1}{c}{$S_{m}$} & $S_{m}{ }^{*}$ \\
\hline$U_{l}$ & $\mu_{\mathrm{eff}}$ & $-\alpha_{l} \frac{\partial P}{\partial x}+\alpha_{l} \rho_{l} g_{x}$ & $-F_{u}$ \\
$U_{g}$ & $\mu_{\mathrm{eff}}$ & $-\alpha_{g} \frac{\partial P}{\partial x}+\alpha_{g} \rho_{l} g_{x}$ & $F_{u}$ \\
$V_{l}$ & $\mu_{\mathrm{eff}}$ & $-\alpha_{l} \frac{\partial P}{\partial r}$ & $-F_{v}$ \\
$V_{g}$ & $\mu_{\mathrm{eff}}$ & $-\alpha_{g} \frac{\partial P}{\partial r}$ & $F_{v}$ \\
$k$ & $\frac{\mu_{t}}{\sigma_{k}}$ & $\alpha_{l} \rho_{l}(G-\varepsilon)+S_{k 1}+S_{k 2}$ & 0 \\
$\varepsilon$ & $\frac{\mu_{t}}{\sigma_{\varepsilon}}$ & $\alpha_{l} \rho_{l} \frac{\varepsilon}{k}\left(C_{1 \varepsilon} G-C_{2 \varepsilon} \varepsilon\right)+S_{\varepsilon 1}+S_{\varepsilon 2}$ & 0 \\
\hline
\end{tabular}

given here.

In essence we write down the turbulent Navier-Stokes equations for both the gas phase and the liquid phase and we couple these through the interface friction coefficients. The relevant equations and the boundary conditions are summarized as follows while the expressions for the terms in the equations are given in Table 1 and further details have been provided in Ilegbusi and Szekely. ${ }^{5)}$ These equations were solved numerically, using a modification of the PHOENICS computational package. ${ }^{6)}$

\subsubsection{Conservation Equations}

The Governing Differential Equation for Phase $m$

The partial differential conservation equations governing the flow of phase $m$ can be represented as follows:

Mass Conservation

$$
\nabla \cdot\left(\alpha_{m} \rho_{m} U_{m}-\rho_{m} \Gamma_{m} \nabla \alpha_{m}\right)=0
$$

where subscript $m$ refers to phase $m, \alpha$ is the volume fraction and $\Gamma$ is the phase diffusion coefficient representing random motion associated with the phases which is expressed as:

$$
\Gamma=\mu_{t} / \rho_{l}
$$

in which $\mu_{t}$ is the turbulent viscosity defined below.

Momentum Conservation

$$
\begin{array}{cl}
\nabla \cdot\left(\alpha_{m} \rho_{m} U_{m} U_{m}-\alpha_{m} D_{m}\right)= & S_{m}+S_{m}{ }^{*} \\
\text { Convection } & \text { Diffusion Source }
\end{array}
$$

where, $S_{m}$ : Within-fluid source terms

$S_{m}{ }^{*}$ : Inter-fluid source terms.

The expressions for these source terms have been given by Ilegbusi and Szekely ${ }^{5}$ (see Table 1) and will not be repeated here.

The diffusion term $D$ may be expressed in tensorial form as:

$$
D=\frac{\partial}{\partial x_{j}}\left(\mu \frac{\partial U_{i}}{\partial x_{j}}-\rho \overline{u_{i} u_{j}}\right)
$$

where $-\rho \overline{u_{i} u_{j}}$ is the turbulent shear stress associated in the present analysis with the continuous phase. It should be remarked that in Eq. (4), the subscripts refer to the components and not the phases. Two approaches have been employed to model the turbulent shear stress. The first employs the basic $k-\varepsilon$ model which presumes isotropy of the turbulence field, thus:

$$
-\rho \overline{u_{i} u_{j}}=\mu_{t} \frac{\partial U_{i}}{\partial x_{j}}
$$

where $\mu_{t}$, the 'turbulent viscosity' is deduced from:

$$
\mu_{t}=C_{\mu} \rho k^{2} / \varepsilon
$$

The turbulence parameters $k$ and $\varepsilon$ are in turn calculated from transport equations similar to Eq. (4). This method again, has been described in an earlier publication. ${ }^{5)}$

The second method is the use of the algebraic stress model of Rodi ${ }^{7)}$ to account for the anisotropy of the turbulence field (as observed from the experimental measurements of Iguchi et al. $\left.{ }^{4}\right)$. Following Rodi, we use the following expression for $-\rho \overline{u_{i} u_{j}}$ (assuming that wall-proximity effects are negligible), thus:

$$
-\rho \overline{u_{i} u_{j}}=\rho k\left[\frac{2}{3} \delta_{i j}+\frac{\left(1-C_{2}\right)\left(\frac{T_{i j}}{\varepsilon}-\frac{2}{3} \delta_{i j} \frac{G}{\varepsilon}\right)}{C_{1}+\frac{G}{\varepsilon}-1}\right] \ldots
$$

where $T_{i j}$ is the stress production term defined as:

$$
T_{i j}=-\overline{u_{i} u_{k}} \frac{\partial U_{j}}{\partial x_{k}}-\overline{u_{j} u_{k}} \frac{\partial U_{i}}{\partial x_{k}}
$$

and $G$ is the rate of production of turbulence defined as:

$$
G=\mu_{t}\left\{\left(\frac{\partial U_{l}}{\partial r}+\frac{\partial V_{l}}{\partial x}\right)^{2}+2\left[\left(\frac{\partial U_{l}}{\partial x}\right)^{2}+\left(\frac{\partial V_{l}}{\partial r}\right)^{2}\right]\right\}
$$

In Eq. (7), $\delta_{i j}$ is the Kronecker delta while $C_{1}$ and $C_{2}$ are empirical coefficients assigned values of 1.8 and 0.6 respectively. More details of this model can be found in Rodi $^{7)}$ and Ilegbusi. ${ }^{8)}$

The boundary conditions are similar to those employed in an earlier publication ${ }^{5)}$ namely;

1) plug gas velocity at the inlet nozzle,

2) zero velocities at solid boundaries imposed through a wall function approach,

3) zero flux accross the central (symmetry) axis, and

4) frictionless free surface that is impervious to water; however, in order to allow the application of a steady solution scheme, gas is allowed to escape at the rate that it arrives at the surface.

\subsection{The Experimental Measurements}

The experimental arrangements and the measurement technique employed have been described fully in previous publications ${ }^{3,4)}$ and therefore only a very brief outline is given here. In essence a cylindrical vessel was employed, into which gas, i.e., air was injected through the bottom in an axi-symmetric arrangement. The bath diameter was $126 \mathrm{~mm}$, bath depth was $233 \mathrm{~mm}$, and the inner diameter of centric nozzle was $2 \mathrm{~mm}$. The gas flow rate ranged from 10.3 to $41.4 \mathrm{~cm}^{3} / \mathrm{s}$.

The actual measurements taken, using laser Doppler 
velocimeter included both the time smoothed and the rms velocity components as well as collecting information on the flow rate of the injected gas.

\section{Comparison of the Measurements and the Predictions}

In the following Figs. 2-7 we shall present a comparison of the measurements and the theoretical predictions.

Figures 2(a) and 2(b) show a comparison of the computed and the experimentally determined velocity vector plots, referring to the upper quadrant of the domain. The velocity vectors in Fig. 2(b) were obtained with the conventional $k-\varepsilon$ model because the mean profile

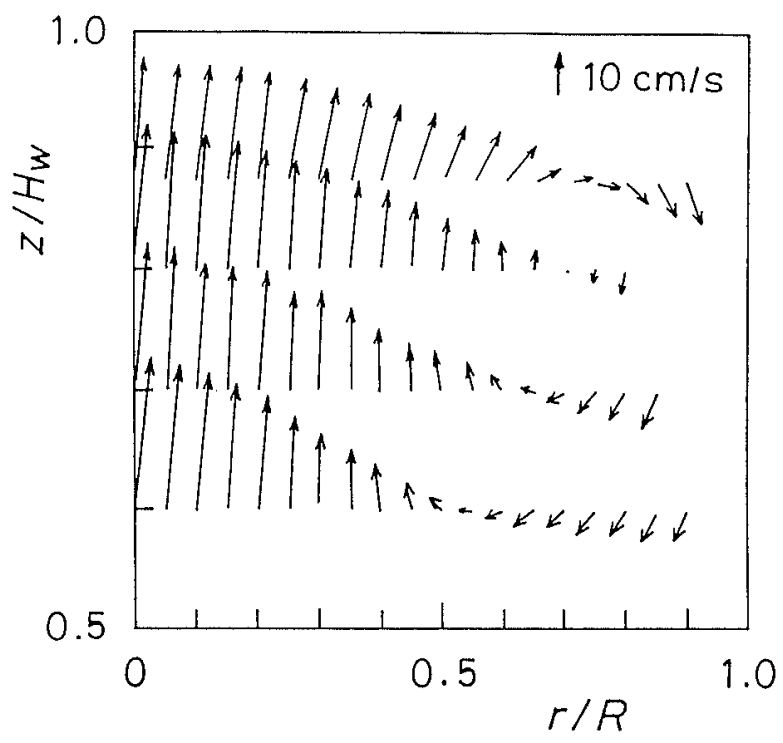

Fig. 2(a). Measured liquid velocity vectors at the upper part of the vessel. (Data of Iguchi et al. ${ }^{4)}$ )

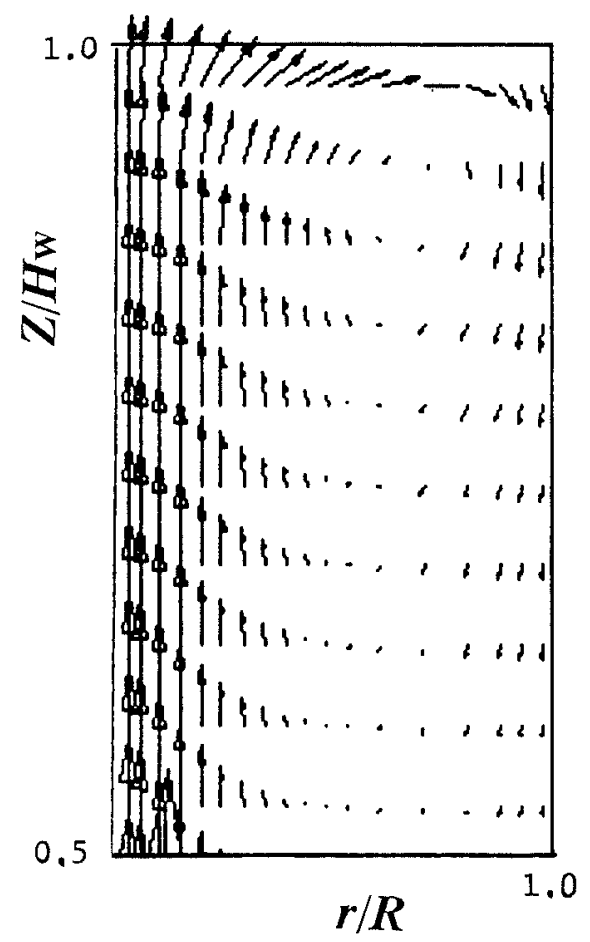

$35 \mathrm{~cm} / \mathrm{s}$
$\uparrow$

Fig. 2(b). Predicted liquid velocity vectors at the upper part of the vessel for the experimental condition of data of Iguchi et al. ${ }^{4)}$ obtained with the algebraic stress model (ASM) was not significantly different from the $k-\varepsilon$ result. It is seen that the agreement is very good, both regarding the absolute values of the velocity vector and its direction.

Figure 3 shows a comparison of the radial variation of the axial and the radial velocity components at a particular height and it is seen that the agreement is again quite reasonable, with the maximum discrepancy being less than about 15 and $10 \%$ for the basic $k-\varepsilon$ model and algebraic stress model respectively. The radial component was quite small, for both the computations and the measurements.

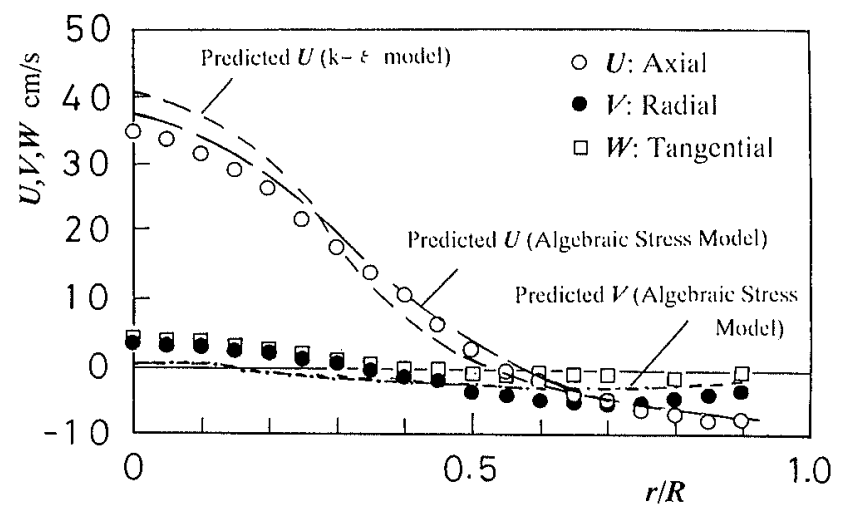

Fig. 3. Mean liquid velocity profiles at $z=139.8 \mathrm{~mm}$. (Data of Iguchi et al. ${ }^{4}$ )

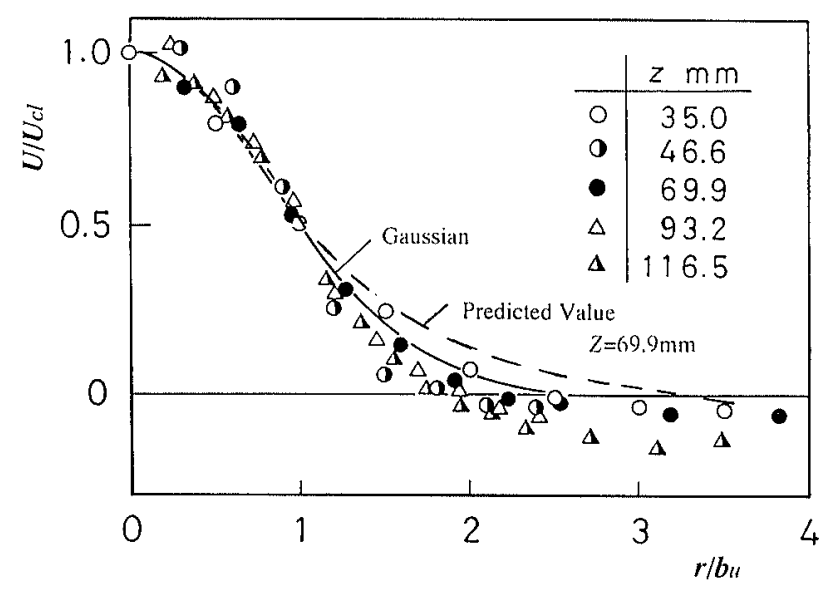

Fig. 4. Radial variation of normalised axial liquid velocity at $z=69.9 \mathrm{~mm}$. (Data of Iguchi et al. ${ }^{4}$ )

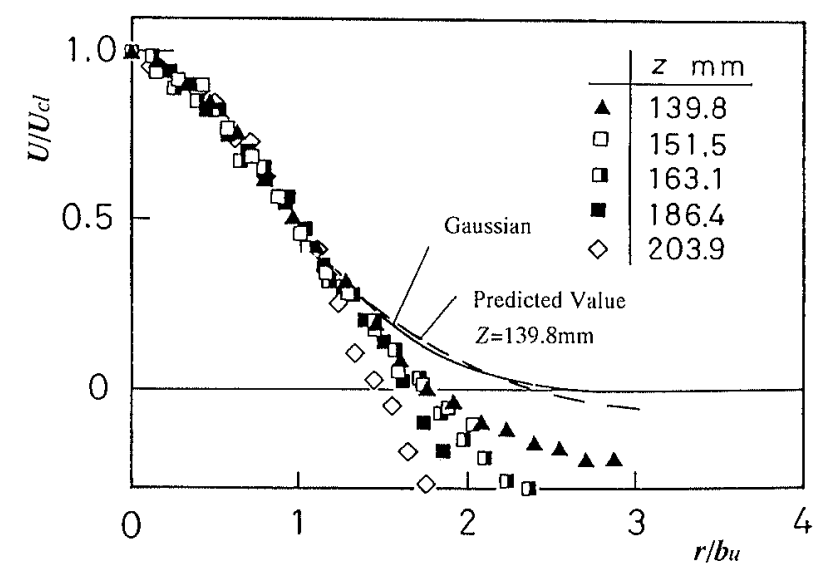

Fig. 5. Radial variation of normalised axial liquid velocity at $z=139.8 \mathrm{~mm}$. (Data of Iguchi et al. ${ }^{4}$ ) 


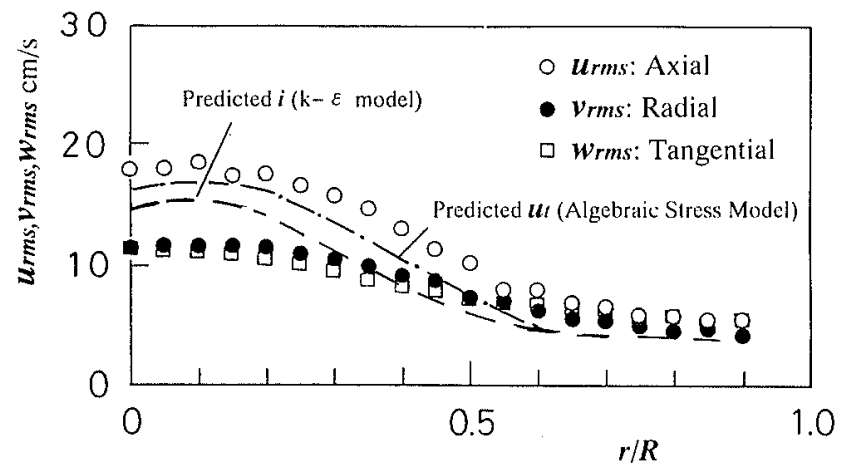

Fig. 6. A comparison of $\mathrm{rms}$ fluctuating liquid velocities measured by Iguchi et al. with predicted rms velocity and 'turbulent velocity'.

The remaining results to be presented are for the algebraic-stress model situation.

Figures 4 and 5 show normalized velocity plots, using the centerline velocity, at 69.9 and $139.8 \mathrm{~mm}$ respectively from the inlet. The horizontal axis in each figure shows the radial coordinate, divided by the half width of the jet. It is seen that the agreement is quite good, except for the very outer region, where the velocities are quite small and where reverse flow occurs. It should be noted that this type of behavior may be expected somewhat, because the flow will be self similar only within and in the vicinity of the plume region.

The experimental work of Iguchi et al. also provided information on the rms velocities. These data are shown in Fig. 6. It is of interest to note that while the rms velocities tend to decay as we proceed from the central axis toward the wall, the results also exhibit anisotropy, that is the fluctuating velocities are greater in the axial direction than in the radial or tangential directions.

One of the present mathematical modelling techniques, using the basic $k-\varepsilon$ model cannot predict such anisotropy; nonetheless, the theoretically predicted "turbulent velocity" $\left[i=(2 \mathrm{k} / 3)^{1 / 2}\right]$ appears to fall between these two limiting cases-at least within the plume region. On the other hand, the rms velocity calculated from the relation:

$$
u_{t}=\left(\overline{u^{2}}\right)^{1 / 2}
$$

obtained from the algebraic stress model appears to agree fairly well with the measured turbulent fluctuation in the axial direction.

Finally, for many practical purposes it would be desirable to predict the melt circulation from a relatively simple correlation, rather than having to resort to complex computations for each specific case.

Two such correlations for the flow rate of water rising upward in the jet region have been recently proposed by Ishigaki ${ }^{9)}$ and Iguchi et $a l .^{4)}$ as follows:

Ishigaki Correlation

$$
Q=4.53 U_{c l} b_{u}^{2}
$$

where $U_{c l}$ is the axial velocity at the axis and $b_{u}$ is the half-value radius based on the mean axial velocity.

Iguchi et al. Correlation

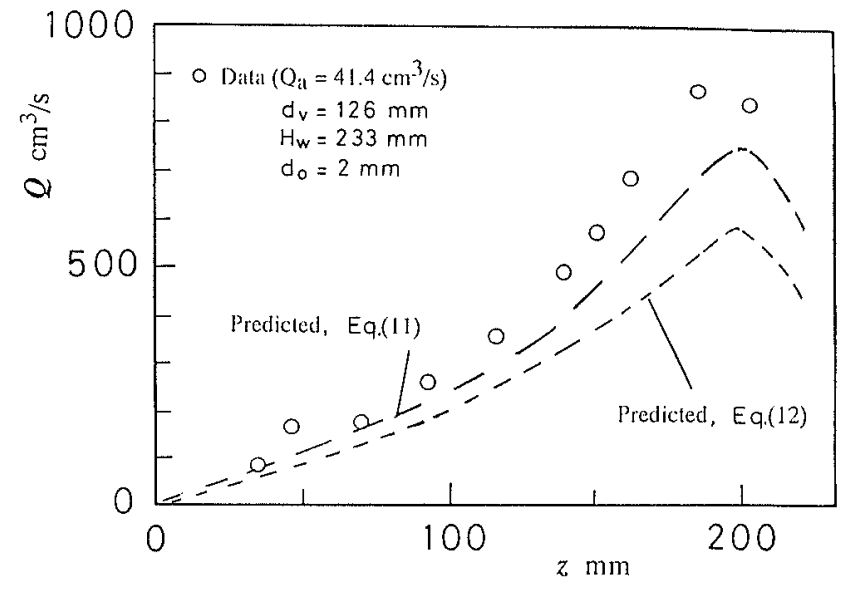

Fig. 7. A comprison of predicted and measured flow rate of rising water induced by bubbles for $Q_{a}=41.4 \mathrm{~cm}^{3} / \mathrm{s}$.

$$
Q=3.6 U_{c l} b_{u}^{2}
$$

Figure 7 shows a plot of the experimental measurements, together with the results of these two correlations. A maximum on each predicted curve was caused by surface stream directed outward. Predictions based on the mathematical model developed here are also shown, for the sake of comparison. It is seen that the agreement is quite reasonable.

\section{Discussion}

The purpose of the paper was to perform a critical comparison between the theoretically predicted velocity profiles and those measured experimentally in a water model of an argon stirred ladle. The main motivation for this work was provided by the fact that both the measurements and the predictions represented the "state of the art" in these systems.

More specifically, the measurements provided for the first time, reliable data on all three velocity components in the system, together with the appropriate turbulence parameters.

As far as the theoretical predictions were concerned, by writing down the Navier-Stokes equations for both the continuous and the dispersed phases, we were able to predict both the plume shapes and the velocity fields from first principles.

The theoretically predicted velocity fields were found to be in good agreement with the measured values. It should be remarked that, in modifying the mathematical model to allow for the anisotropy, the actual agreement was significantly improved. This modification of the $k-\varepsilon$ model, which has been described in the paper, may have considerable utility, because it allows the modelling of anisotropic systems without the need to resort to direct simulation or large eddy simulation, thus avoiding the significantly increased computational labor.

The approach outlined in the paper shows clear promise for tackling a broad class of two phase problems, including multi-plume systems and possibly an extension to three phase situations.

In conclusion it may be appropriate to list the as yet unresolved problems, which include the following: 
By using time smoothed velocities, of course one could not capture the unsteady nature of the flow; the solution of this problem will have to await the adaptation of direct simulation methods to these situations.

In the model no allowance has been made for the formation of the dome, although a correction has been made by allowing the escape of gas from the plume region, thus minimizing the artificial recirculation.

Finally no allowance has been made for free surface disturbances or slopping, which would require a sophisticated free surface capability.

These and other issues will be addressed in a future publication.

\section{Nomenclature}

$b_{u}$ : Half value radius of axial mean velocity

$d_{v}:$ Vessel diameter

$d_{0}:$ Nozzle diameter

$D$ : Effective diffusivity

$G$ : Generation rate of turbulence energy

$H_{w}$ : Height of liquid in vessel

$i$ : Turbulent velocity

$k$ : Turbulence kinetic energy

$P$ : Static pressure

$Q:$ Flow rate of water rising in plume

$Q_{a}$ : Gas flow rate

$r, z$ : Radial and axial coordinates

$S$ : Within-fluid source terms

$S^{*}:$ Inter-fluid source terms

$T_{i j}$ : Stress-production term

$u, v, w$ : Fluctuating velocities along $x, r, \theta$ directions respectively $u_{\mathrm{rms}}, v_{\mathrm{rms}}, w_{\mathrm{rms}}: \quad$ Measured rms values of $u, v, w$, respectively

$U, V, W$ : Mean velocity along $x, r, \theta$ direction respectively

$U_{g}, U_{l}$ : Gas velocity, liquid velocity in axial direction

$V_{g}, V_{l}$ : Gas velocity, liquid velocity in radial direction

$u_{t}$ : Calculated $\mathrm{rms}$ velocity in axial direction (Eq. (10))

$\varepsilon:$ Rate of dissipation of turbulence energy

$\rho$ : Density

$\mu:$ Laminar viscosity

$\mu_{t}$ : Turbulent viscosity

\section{REFERENCES}

1) A. H. Castillejos and J. K. Brimacombe: Scaninject IV, Part I, No. 16, 4th Int. Conf. on Injection Metallurgy, Lulea, Sweden, (1986), 16.1.

2) A. H. Castillejos: "A study of the fluid dynamic characteristics of turbulent gas-liquid plumes", Ph.D. Thesis, Dept. of Metallurgical Engineering, University of British Columbia, Canada, (1986).

3) M. Iguchi, J. Tani, T. Uemura, H. Kawabata and H. Takeuchi: Tetsu-to-Hagané, 74 (1988), 1785.

4) M. Iguchi, H. Takeuchi and Z. Morita: ISIJ Int., 31 (1991), 246.

5) O. J. Ilegbusi and J. Szekely: ISIJ Int., 30 (1990), 731.

6) D. B. Spalding: Mathematics and Computers in Simulation, XIII, (1981), 267.

7) W. Rodi: Turbulence Models and Their Application in Hydraulics-A State of the Art Review, IAHR Delft, The Netherlands, (1980).

8) O. J. Ilegbusi: Appl. Math. Model., 9 (1985), 263.

9) H. Ishigaki: Trans. Jpn. Soc. Mech. Eng., 48B (1982), 1692. 